\title{
Treatment Compliance Communications Between Patients with Severe Mental Illness and Treating Healthcare Providers: A Retrospective Study of Documentation Using Healthcare Reimbursement Claims and Medical Chart Abstraction
}

\author{
Carolyn Martin' \\ Eleena Koep' \\ John White' \\ Angela Belland' \\ Heidi Waters (iD ${ }^{2}$ \\ Felicia Forma (iD ${ }^{3}$ \\ 'Optum, Eden Prairie, MN, USA; ${ }^{2}$ Policy \\ Research Health Outcomes, Otsuka \\ Pharmaceutical Development \& \\ Commercialization, Inc, Princeton, NJ, \\ USA; ${ }^{3}$ Otsuka Pharmaceutical \\ Development \& Commercialization, Inc., \\ Health Economics and Outcomes \\ Research, Princeton, NJ, USA
}

\begin{abstract}
Introduction: Successful treatment for serious mental illnesses (SMIs) requires a good therapeutic alliance with healthcare providers and compliance with prescribed therapies such as antipsychotic medications. This retrospective study, which utilized administrative claims linked with abstracted medical chart data, addressed a data gap regarding compliance-related discussions between providers and patients.
\end{abstract}

Methods: Commercially insured patients in ambulatory care post-acute (emergency or inpatient) event were eligible. Criteria included age 18-65 years; schizophrenia, bipolar disorder, or major depressive disorder diagnoses; continuous enrollment 6 months before to 12 months after the first acute event claim dated $01 / 01 / 2014$ to $12 / 31 / 2015$; and antipsychotic medication prescription. Demographic and clinical data, and patient-provider discussions about treatment compliance were characterized from claims and abstracted medical charts.

Results: Ninety patients (62\% female, mean age 41 years) were included and 680 visits were abstracted; only 58\% had first-visit antipsychotic compliance discussions. Notably, $18 \%$ of patients had discussions using the specific terms "compliance," "persistence," or "adherence," whereas half were identified by more general terms. Compliance discussions were observed least often among the patients with schizophrenia, as compared with bipolar or major depressive disorders - a counterintuitive finding.

Discussion: Compliance discussions may represent intervention opportunities to optimize treatment, yet their study is a complex endeavor. The results of this study show an opportunity to improve this valuable treatment step.

Keywords: schizophrenia, bipolar disorder, major depression disorder, medication compliance, antipsychotic medication

\section{Introduction}

Schizophrenia, bipolar disorder, and major depressive disorder are characterized as severe mental illnesses (SMIs), which contribute substantially to poor health, disability, and premature mortality, as well as a tremendous economic burden. ${ }^{1}$ Globally, annual direct healthcare and indirect costs for mental illness have been projected to be $\$ 6$ trillion by $2030 .^{2}$ As of 2017 , an estimated 11 million adults were living with SMIs,
Correspondence: Carolyn Martin

Optum, I 1000 Optum Circle, Eden

Prairie, MN, 55344, USA

Tel + I 952-205-7716

Fax +I 8775037362

Email carolyn.martin@optum.com 
yet one-third did not receive mental health treatment in the prior year. ${ }^{3}$ Estimates of the US burden have been $\$ 210$ billion for major depressive disorder, ${ }^{4} \$ 156$ billion for schizophrenia, ${ }^{5}$ and $\$ 200$ billion for bipolar disorder, ${ }^{6}$ per year.

Successful treatment for SMIs involves effective therapeutic relationships with healthcare providers, patient selfengagement in managing the condition, and adherence to a treatment regimen, which often includes antipsychotic medications. ${ }^{7-9}$ However, non-adherence to antipsychotic medications has been reported at 50-70\%. ${ }^{10-12}$ Many measures validate the impact of non-adherence: higher risks of relapse, costly inpatient and emergency care, and societal economic burden, and decline in patient overall health and quality of life. ${ }^{13-18}$

The reasons for non-adherence are varied and personal, including side effects, negative attitude toward medication, poor insight, and cognitive impairment. ${ }^{19-22}$ Although some factors are outside the control of patients or their providers, studies have shown that a sense of therapeutic alliance can reduce intentional non-adherence. ${ }^{14,19,23,24}$ Establishing a good clinician-patient collaborative relationship may improve patient attitudes toward medication and psychiatric care. ${ }^{25-27}$

Thus, initial and ongoing patient-provider interactions represent points in the care spectrum at which targeted interventions may improve outcomes via improved adherence. ${ }^{28}$ Yet, assessing adherence during outpatient care is difficult. ${ }^{27}$ Data are scarce describing dialogues regarding compliance with antipsychotic medication or other treatments. ${ }^{15}$ The purpose of this study was to describe the frequency and circumstances of communications between providers and patients regarding treatment compliance among patients with symptomatic SMI.

\section{Methods}

\section{Study Design and Data Sources}

This was a retrospective study conducted using administrative data from the Optum Research Database (ORD) linked with abstracted medical chart data to form a patient-level analytic dataset that included both administrative and clinical data. The ORD is geographically diverse across the United States (US) and contains de-identified medical and pharmacy claims data and enrollment information for insured individuals.

Data were accessed in compliance with US data protection and privacy regulations. The study protocol and chart data collection form were reviewed and approved by the New England Institutional Review Board (\#120170201), including a waiver of informed consent.

\section{Claims-Based Sample Selection}

Commercially insured patients were identified by evidence of an acute (inpatient hospitalization or emergency department [ED] visit) behavioral health $(\mathrm{BH})$ treatment claim dated from 01 January 2014 through 31 December 2015 (identification period). The date of the first acute event during the identification period was defined as the index date (Figure 1). Claims data were extracted for the 6-month (baseline) period before the index date. The follow-up period was 12 months post-index for claims data, and up to 12 months after the first abstracted office visit for medical chart data.

Inclusion criteria were as follows: claims-based evidence of an ED visit or inpatient hospitalization (index $\mathrm{BH}$ visit) with a diagnosis code (in any position) for schizophrenia, bipolar disorder (I or II), or major

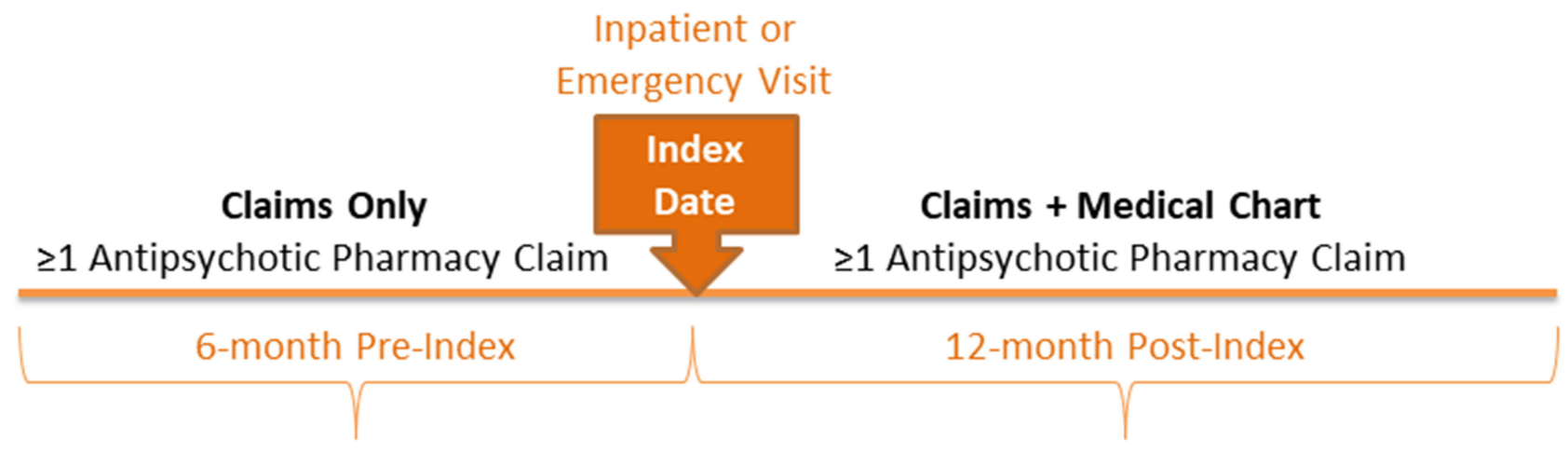

Figure I Study design. 
depressive disorder during the identification period (Appendix Table A1); age 18-65 years at the index date; complete demographic and insurance information available; continuous health insurance coverage with medical and pharmacy benefits during the baseline and follow-up periods (meaning all healthcare claims submitted for reimbursement by insurance were available for inclusion in the study data); and at least one ambulatory visit for $\mathrm{BH}$ treatment within 90 days of discharge. Age restrictions reflect exclusion of minor patients who would have care managed by a parent or guardian and patients older than 65 who were less likely commercially insured. Furthermore, included patients had at least 1 pharmacy claim for antipsychotic medication (Appendix Table A2) during the baseline period and within 60 days after the index date (minimum of 2 claims). Claims were not required to be for the same medication. These criteria were selected to identify patients with more severe disease or difficult to treat symptoms for whom treatment compliance may be critical to prevent acute events.

Patients were excluded if there was no evidence of follow-up ambulatory treatment with a primary diagnosis of interest. Patients were excluded by claims for substance abuse treatment during baseline or evidence of substance abuse treatment (Appendix Table A3) in the medical chart up to 90 days pre-index. This step helped select patients for whom SMI was the primary focus of treatment. Finally, patients with residential $\mathrm{BH}$ treatment after the index event were excluded because medication adherence would have been controlled by the treatment program. Patients meeting the inclusion criteria and each patient's treating provider were identified to facilitate collection of chart-based measures from the patient's medical chart of interest.

\section{Claims-Based Measures}

Demographic measures obtained from claims included age as of index date, sex, and US Census geographic location. ${ }^{29}$ Clinical data included baseline comorbid conditions and $\mathrm{BH}$ diagnoses, and $\mathrm{BH}$ medication use during baseline and follow-up. The most common comorbid conditions were identified using Clinical Classifications Software from the Agency for Healthcare Research Quality. ${ }^{30}$ Baseline comorbidity burden was characterized by the Quan-Charlson comorbidity score. ${ }^{31}$

Baseline and follow-up BH-related healthcare resource utilization (HCRU) was calculated for ambulatory visits, ED visits, and inpatient admissions. BH-related visits and admissions were identified from claims with a $\mathrm{BH}$-related diagnosis code (ie, attention-deficit/hyperactivity disorders, adjustment disorders, anxiety disorders, bipolar disorder, depressive disorders, personality disorders, and schizophrenia) in the primary diagnosis position. Claims with diagnosis codes for cognitive (eg, Alzheimer disease and other dementias) and/ or developmental disorders were excluded. BH-related medication use was identified from claims for antidepressants, antipsychotics, mood stabilizers, and antianxiety medications [Appendix Table A2]. BH-related healthcare costs were calculated using claims with a $\mathrm{BH}$ diagnosis code in the primary position. Costs included health insurer- and patient-paid amounts adjusted to 2017 US dollars using the Consumer Price Index. $^{32}$

\section{Chart Abstraction and Cohort Assignment}

Healthcare providers of patients meeting the study criteria were targeted for participation in the medical chart abstraction process. Prescribing providers, with a focus on psychiatry and neurology specialists, were prioritized for participation, followed by other clinical specialties. Provider site of care identifying information was captured from the administrative claims data. Medical charts, supplied by providers who agreed to participate, were abstracted. Provider participation was voluntary and not all providers contacted opted to participate. Some charts included treatment notes by collaborating non-prescriber clinicians. Only ambulatory visits that included BH treatment were abstracted. Starting from the first ambulatory visit with a $\mathrm{BH}$ diagnosis post-index, all available visits through 12 months of follow-up were abstracted.

Patients were assigned to study cohorts (schizophrenia [SZ], bipolar disorder [BD], or major depressive disorder [MDD]) based on the $\mathrm{BH}$ diagnosis on the first abstracted office visit. Patients whose first visit contained more than 1 diagnosis were assigned to the cohort with the highest perceived severity, according to the following hierarchy (patients were not represented in more than one cohort):

(i) Patients with a schizophrenia diagnosis were assigned to the SZ cohort;

(ii) Patients with a bipolar disorder diagnosis and no schizophrenia diagnosis were assigned to the $\mathrm{BD}$ cohort;

(iii) Patients with a major depressive disorder diagnosis and no diagnosis of schizophrenia or bipolar disorder were assigned to the MDD cohort. 


\section{Chart-Based Measures}

For each BH-related office visit, data abstracted from medical charts included the reason for the visit, diagnoses, treatment discussed (antipsychotic medication or other therapies), treating provider specialty (MD, nurse practitioner, other; could have more $>1$ provider type per visit), consultation with other providers, the occurrence and type(s) of change(s) to treatment regimen, and instructions to return for a future visit.

Data abstraction also included sources of psychosocial stress that might impact compliance (eg, physical violence, substance use/abuse, change in housing, prescription drug affordability, job loss or other employment issues, family/ domestic issues, death in family, other, or none) or case management/social interventions.

Compliance-related discussions were identified by reviewing each available medical chart for predetermined specific words ("compliance," "persistence," and "adherence") as well as non-specific words and phrases identified as relating to compliance, persistence, and adherence (eg, "continue taking medications" or "take medications as directed"). For purposes of the study, the term compliance was used broadly to incorporate all terms related to following a prespecified or proscribed treatment. Observed compliance discussions were stratified by topic: compliance with antipsychotic medications versus other interventions (such as individual or group talk therapy). Categories describing type of discussions were not mutually exclusive. Discussion characteristics were reported for those observed in the first visit alone, and for all visits combined, to determine if providers discussed compliance at a first visit after an acute care event, as recommended, ${ }^{33}$ but also whether these discussions were continued over time.

\section{Statistical Analysis}

Patient characteristics and outcomes were stratified by study cohort: SZ, BD, or MDD. Numbers and percentages were provided for categorical variables and means and standard deviations (SDs) were provided for continuous variables. Between-cohort differences in patient characteristics and study outcomes were analyzed using $t$-tests and Fisher Exact tests as appropriate.

A generalized linear model (GLM; binomial distribution, logit link) was constructed to analyze the likelihood of patients having an antipsychotic medication compliance-related discussion (using either compliance-specific or non-specific terms) with their healthcare provider during follow-up. The presence of a compliance discussion was defined as a binary dependent variable. The model was adjusted for repeated measures, as well as for patient demographic characteristics, chart-based BH diagnoses, claims-based baseline clinical characteristics, and number of days between the index date and the first office visit. Odds ratios (ORs), 95\% confidence intervals (CIs), and p-values were calculated for each independent variable. All analyses were conducted using SAS version 9.4 (SAS Institute Inc., Cary, NC).

\section{Results}

\section{Sample Selection and Cohort Assignment}

Among 755 patients who met the inclusion criteria, a stratified sample of 300 patients was identified for medical chart abstraction (Figure 2). The sample included all patients identified with a diagnosis of schizophrenia $(\mathrm{n}=$ 77) from the claims data. Additional patients were randomly selected as a function of the distribution of the BD and MDD diagnoses in the remaining sample, yielding 120 patients with $\mathrm{BD}$, and 103 with MDD. Of the 300 identified for chart procurement, 90 providers chose to participate by providing a chart to be abstracted. Demographic characteristics did not differ $(\mathrm{p}>0.05)$ between patients with eligible medical charts and those who were identified but no chart was procured ( $\mathrm{p}>0.05$ for all). The cohort sizes for the analytic sample were $\mathrm{SZ}=17, \mathrm{BD}=47$, and $\mathrm{MDD}=26$, based on diagnoses abstracted from the firstvisit medical charts among patients whose providers participated in the study.

\section{Patient Characteristics}

Demographics and comorbidity data were sourced from claims. Overall, more than half $(62 \%)$ of patients included in the final analytic dataset were female, with a mean age of approximately 41 years (Table 1). Patients predominantly resided in the South and Midwest regions of the United States, consistent with the database population. The most common baseline comorbid conditions were anxiety disorders, hypertension, and diabetes, observed among $47 \%, 26 \%$, and $22 \%$ of all patients, respectively, with no significant differences by cohort. However, diagnoses of mood disorders (including BD and MDD) and schizophrenia varied by cohort (mood disorders among $98 \%$ of the BD and $85 \%$ of the MDD cohorts, $\mathrm{p}=0.001$; and schizophrenia among $76 \%$ of the SZ cohort, $\mathrm{p}<0.001$ ), although 
BH-related inpatient or ED claims (date of first claim = index) containing diagnoses codes for schizophrenia, bipolar disorder, or major depressive disorder from 01 Jan

$$
2014-31 \text { Dec } 2015
$$

$$
\mathrm{N}=28,569
$$

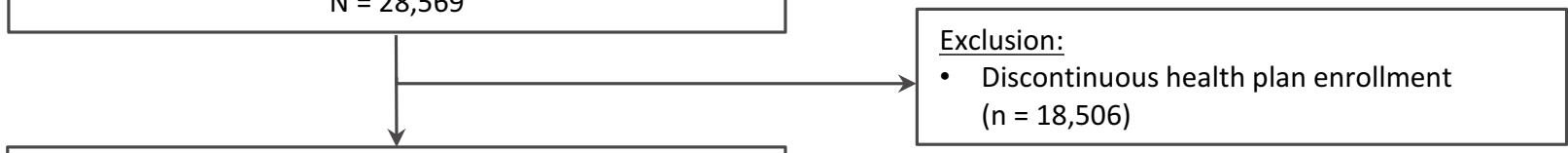

Age $\geq 18$ and $\leq 65$, no missing demographics or insurance information, and continuous enrollment with medical and pharmacy benefits during baseline and follow-up periods $n=10,063$

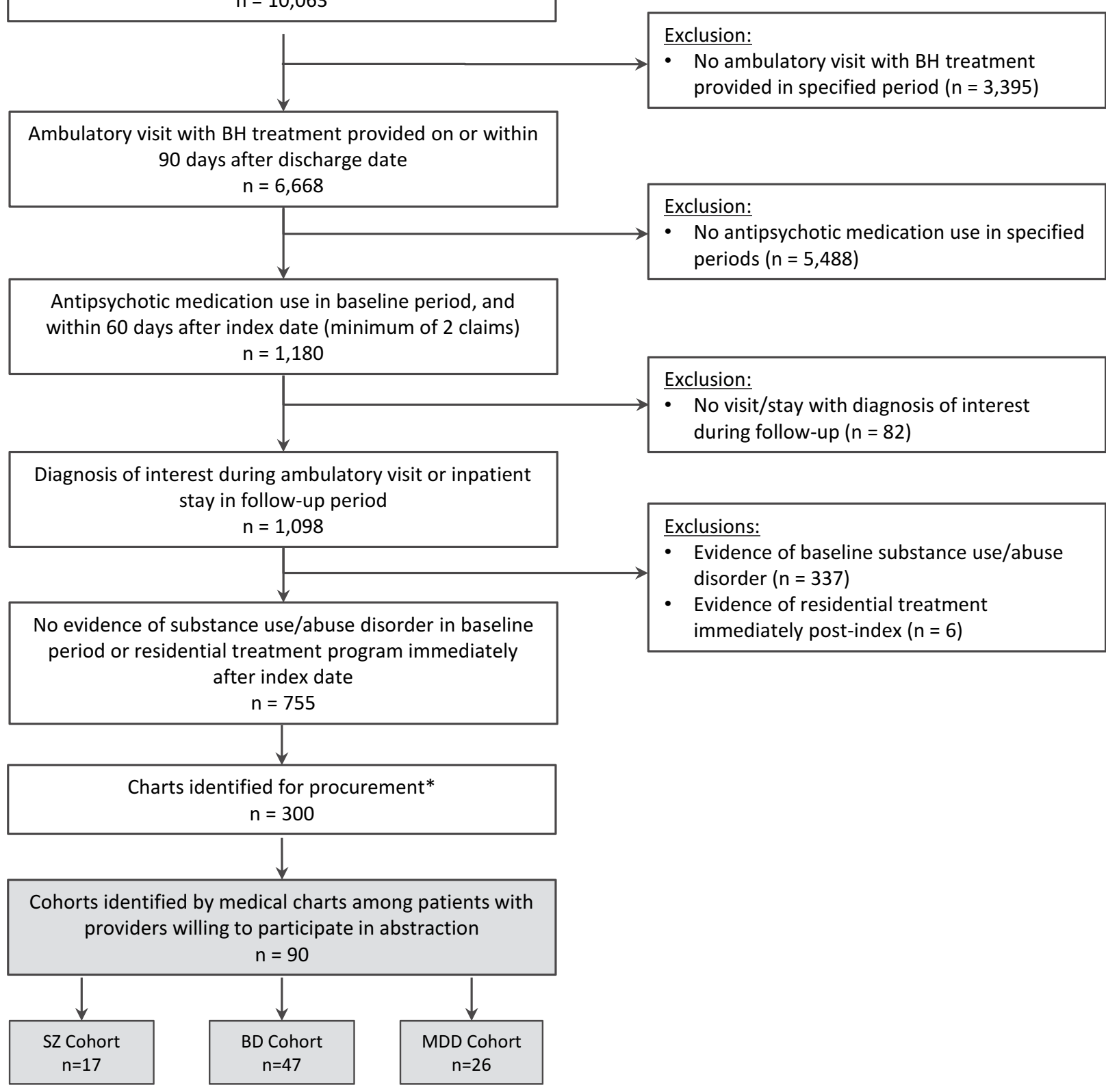

Figure 2 Patient selection and attrition.

Notes: *All patients with schizophrenia identified in the claims data $(n=77)$ were included at this step. The remaining sample was selected from patients with a diagnosis of bipolar disorder $(n=120)$ or major depressive disorder $(n=103)$ observed in chart review, according to cohort assignment hierarchy (see Methods). Patients with $>1$ diagnosis were not included twice.

Abbreviations: BD, bipolar disorder; BH, behavioral health; ED, emergency department; MDD, major depressive disorder; SZ, schizophrenia. 
Table I Patient Demographic and Clinical Characteristics ${ }^{\mathrm{a}}$

\begin{tabular}{|c|c|c|c|c|c|}
\hline Characteristics & Total $(\mathrm{N}=90)$ & $S Z(n=17)$ & $B D(n=47)$ & $\operatorname{MDD}(n=26)$ & p-value \\
\hline \multicolumn{6}{|l|}{ Baseline Claims Findings } \\
\hline Age, years, mean (SD) & $40.8(14.4)$ & $36.1(14.5)$ & $41.3(14.1)$ & $42.8(14.7)$ & 0.303 \\
\hline Female, n (\%) & $56(62)$ & $8(47)$ & $27(57)$ & $2 I(8 I)$ & 0.053 \\
\hline \multicolumn{6}{|l|}{ Geographic region, n (\%) } \\
\hline Northeast & $7(8)$ & I (6) & $3(6)$ & $3(12)$ & 0.868 \\
\hline Midwest & $28(3 I)$ & $6(35)$ & $10(2 I)$ & $12(46)$ & 0.071 \\
\hline South & $44(49)$ & $9(53)$ & $26(55)$ & $9(35)$ & 0.237 \\
\hline West & $11(12)$ & I (6) & $8(17)$ & $2(8)$ & 0.469 \\
\hline Baseline Quan-Charlson comorbidity score, mean (SD) & $0.3(0.8)$ & $0.1(0.2)$ & $0.4(1.0)$ & $0.3(0.6)$ & 0.277 \\
\hline \multicolumn{6}{|l|}{ Most common baseline comorbid conditions, ${ }^{b}$ n (\%) } \\
\hline Mood disorders & $79(88)$ & $11(65)$ & $46(98)$ & $22(85)$ & 0.001 \\
\hline Anxiety disorders & $42(47)$ & $6(35)$ & $20(43)$ & $16(62)$ & 0.182 \\
\hline Schizophrenia and other psychotic disorders & $24(27)$ & $13(76)$ & $7(15)$ & $4(15)$ & $<0.001$ \\
\hline Hypertension & $23(26)$ & $3(18)$ & $12(26)$ & $8(31)$ & 0.690 \\
\hline Other connective tissue disease ${ }^{c}$ & $23(26)$ & $2(12)$ & $13(28)$ & $8(31)$ & 0.361 \\
\hline Disorders of lipid metabolism & $21(23)$ & $6(35)$ & II (23) & $4(15)$ & 0.336 \\
\hline Respiratory infections & $21(23)$ & $2(12)$ & $14(30)$ & $5(19)$ & 0.317 \\
\hline Diabetes mellitus without complications & $20(22)$ & $4(24)$ & $10(2 I)$ & $6(23)$ & 1.000 \\
\hline Other nutritional, endocrine, and metabolic disorders & $18(20)$ & $3(18)$ & $7(15)$ & $8(31)$ & 0.295 \\
\hline Count of unique BH-related diagnoses, ${ }^{\mathrm{d}}$ mean (SD) & $\mathrm{I} .4(0.8)$ & $1.4(0.8)$ & $1.5(0.7)$ & $1.3(0.8)$ & 0.590 \\
\hline \multicolumn{6}{|l|}{ First-visit chart abstraction findings } \\
\hline Patients with psychosocial stress noted, $\mathrm{n}(\%)$ & $61(68)$ & $12(7 \mid)$ & $28(60)$ & $2 I(8 I)$ & 0.181 \\
\hline \multicolumn{6}{|l|}{ Type of psychosocial stress, n (\%) } \\
\hline Family/domestic issues & $36(59)$ & $6(50)$ & $18(64)$ & $12(57)$ & 0.664 \\
\hline Job loss, other employment issues & $21(34)$ & $4(33)$ & $9(32)$ & $8(38)$ & 0.938 \\
\hline Other ${ }^{e}$ & $18(30)$ & $3(25)$ & $8(29)$ & $7(33)$ & 0.874 \\
\hline Death in family & $5(8)$ & I (8) & I (4) & $3(14)$ & 0.413 \\
\hline Physical violence & $5(8)$ & I (8) & $4(14)$ & $0(0)$ & 0.200 \\
\hline
\end{tabular}

Notes: ${ }^{a}$ Numbers and percentages were provided for categorical variables and means and standard deviations (SDs) were provided for continuous variables. Betweencohort differences in patient characteristics and study outcomes were analyzed using $t$-tests and Fisher Exact tests as appropriate. 'ldentified from claims using Clinical Classifications Software from the AHRQ. ${ }^{30}$ Includes conditions such as tendinitis, bursitis, and presence of artificial limbs or joints. ${ }^{\mathrm{d} B a s e d}$ on administrative claims with a $\mathrm{BH}$ diagnosis code in the primary position during the baseline period. ${ }^{\mathrm{e}}$ Includes financial, physical health, school-related, and legal stress; visa issues, boredom, and other mental health stress.

Abbreviations: AHRQ, Agency for Healthcare Research and Quality; BH, behavioral health; SD, standard deviation.

many patients had claims evidence of more than 1 diagnosis prior to the index event. Chart abstraction at the first post-index visit indicated half of the patients had more than $1 \mathrm{BH}$ diagnosis $(53 \%$; 48/90). Among patients with more than one diagnosis, $90 \%$ (43/48) had diagnoses other than $\mathrm{SZ}, \mathrm{BD}$, or MDD, most commonly anxiety disorders (30 of $43 ; 70 \%$ ), and attention deficit-hyperactivity/attention deficit disorders (14 of 43; 33\%) (data not shown).

Among patients whose first-visit charts indicated any (61 of 90;68\%) psychosocial stress surrounding the time of the index acute event, 59\% (36 of 61) indicated family/ domestic issues were a source (Table 1). However, the patient's living arrangement was not well documented: only $19 \%$ of first-visit charts included residential setting.

\section{Claims-Identified BH Healthcare and Medication Use}

Findings for HCRU were consistent with expectations. Most patients (88\%) had at least 1 baseline claim for BHrelated ambulatory care, with a higher proportion observed for the BD cohort ( $\mathrm{p}=0.015)$. Overall, $32 \%$ had at least one 
BH-related ED visit, and 16\% had at least one BH-related inpatient admission during baseline. These patients had high HCRU during both the baseline and follow-up periods, with a mean (SD) total BH-related healthcare cost of $\$ 5295$ (\$7264) during baseline and $\$ 15,121 \quad(\$ 16,635)$ during follow-up.

The distribution of $\mathrm{BH}$ medications was also as expected for SMI cohorts. After antipsychotic medications, benzodiazepines were the most common: 58\% of patients had $\geq 1$ pharmacy claim in baseline and follow-up periods. During baseline, the next most commonly filled medications were selective serotonin reuptake inhibitor (SSRI) antidepressants (43\% of all; highest among MDD cohort; $\mathrm{p}<0.001)$ and mood stabilizers $(41 \%$ of all; highest among the BD cohort; $\mathrm{p}<0.001)$. During the follow-up period, SSRI antidepressants (44\% of all) and mood stabilizers $(52 \%$ of all) remained the most common among the entire sample, yet the SZ cohort had the highest proportion of patients with at least one fill $(71 \%$; $<0.001)$ of SSRI antidepressants.

\section{Chart Abstraction Findings}

The mean (SD) time from the end of the index event to the first qualifying abstracted visit was 36.1 (52.2) days overall (53.7 [75.7] for SZ, 25.2 [27.5] for BD, and 44.4 [64.3] for MDD cohorts; $p=0.097$ ). The mean (SD) number of abstracted office visits was 7.6 (4.4) per patient over the 12-month follow-up period, with no significant differences across cohorts (7.9 [4.6] for SZ, 7.8 [4.0] for BD, and 6.9 [5.2] for MDD cohorts; $\mathrm{p}=0.663$ ).

A change in $\mathrm{BH}$ symptoms prompted $67 \%$ of first abstracted visits. While psychotropic medication use was discussed in nearly all (96\%) of the first visits, only half of patients' first visits included a discussion of behavioral health talk therapies (53\% for SZ, $45 \%$ for $\mathrm{BD}$, and $58 \%$ for MDD cohorts) (Table 2). When all visits were analyzed, the proportion that included discussion of talk therapies was lower $(39 \%$ overall $)$ and did not differ statistically by cohort. Most treatment discussions were held with an MD (84\% of first visits, $81 \%$ of all visits); nurse practitioners were the second most prevalent provider type (7\% of first visits, $8 \%$ of all visits). Treatment was changed during follow-up for about half of patients at the first visit. Across all visits, the frequency of medication changes differed by cohort ( $\mathrm{p}=0.009$ ): $40 \%, 55 \%$, and $50 \%$ of visits for the SZ, BD, and MDD cohorts, respectively.
Despite the frequent changes in treatment, not all visits included direction that the patient should return to the provider. At the first visit, $74 \%$ of patients were told to return to the provider. When all visits were considered, this number increased to $84 \%$ overall, but patients in the MDD cohort were less likely (76\%) to be told to return than those in the SZ $(81 \%)$ or BD $(90 \%)$ cohorts $(\mathrm{p}<$ 0.001).

Furthermore, among patients who were told at the first visit to return to the provider, the indicated time period was inconsistent (Figure 3), although differences were not significant between cohorts. Nearly all patients were instructed to return within 3 months $(96 \%$ at the first visit; 98\% among all visits).

\section{Observations of Compliance Discussions}

At the first abstracted visit, fewer than 1 in 5 charts showed compliance discussions using the predetermined specific terms ("compliance," "persistence," and "adherence") (Figure 4). More commonly, non-specific terms were observed: 49\% (44/90) of entire sample, 41\% (7/ 17) of SZ, $60 \%$ (28/47) of BD, and 35\% (9/26) of MDD cohorts $(\mathrm{p}=\mathrm{ns})$ had discussion using non-specific terms. In general, with specific or non-specific terms, compliance discussions were less common for non-medication treatments at the first visit.

Identification of compliance discussions among all visits abstracted also differed considerably by specific terms versus non-specific terms. The mean (SD) proportion of all visits for which any compliance discussion was documented was 0.63 (0.38): $0.52(0.42)$ in the SZ cohort; 0.69 (0.34) in the BD cohort; and $0.61(0.40)$ in the MDD cohort $(p=0.259)$. Yet, despite having nearly 8 office visits abstracted per patient over 1 year, fewer than 2 of those (mean $=1.6)$ included a compliance-related discussion using specific terms, whereas over half (mean $=4.3$ ) included a compliance-related discussion using nonspecific terms. These categories (specific v. non-specific) were not mutually exclusive; visits that included both types of terms were counted in each category, but nonspecific terms were more frequently observed. Across all visits, patients in the $\mathrm{BD}$ cohort had more antipsychotic medication compliance-related discussions documented in medical charts, but this was true only for discussions using non-specific terms (91\% of SZ; $93 \%$ of BD, $81 \%$ of MDD cohorts; $\mathrm{p}=0.007$; Table 3).

Compliance discussions were more commonly observed for antipsychotic medication than for non- 


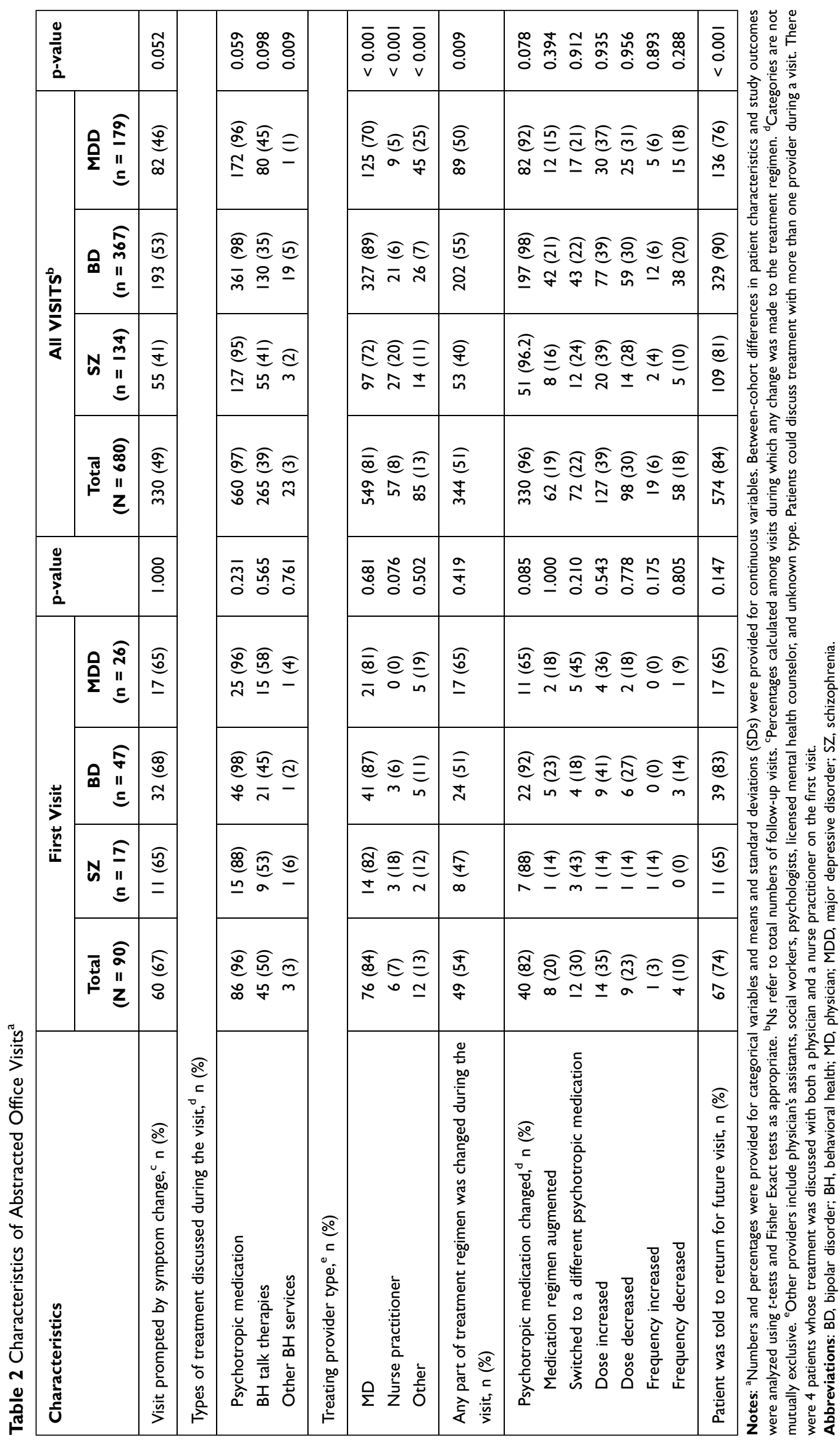




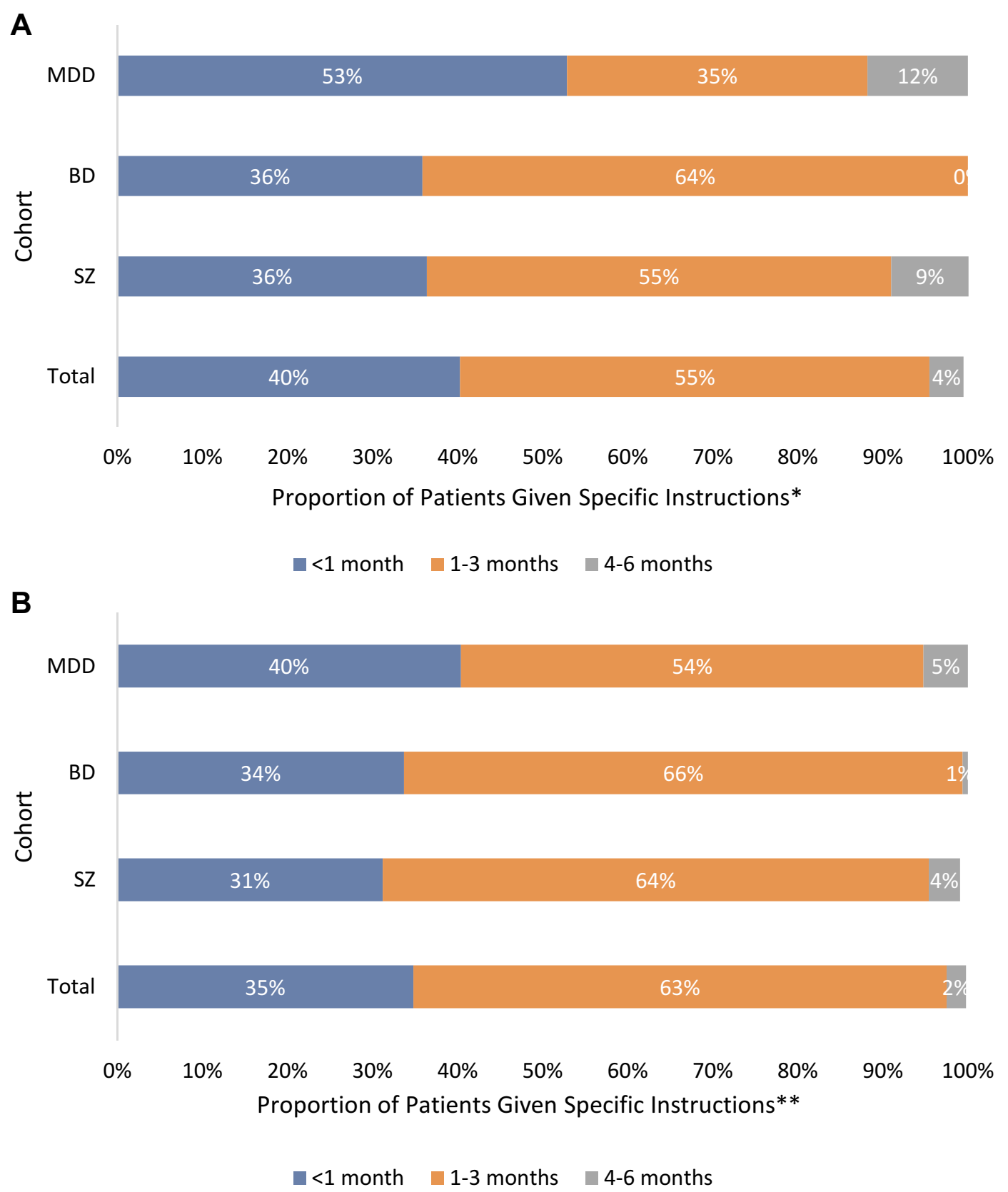

Figure 3 Specific time periods given for return visit at (A) first visit,* and among (B) all visits**. (A) At first visit: *Among number of first visits with documented instructions to return (Total=67; SZ=II; BD=39; MDD=17). Due to rounding of individual values, the total may not add to $100 \%$. (B) Among All Visits: **Among number of all visits with documented instructions to return (Total=574; $S Z=109 ; B D=329 ; M D D=136$ ). Due to rounding of individual values, the total may not add to $100 \%$.

medication BH treatments. Among all visits, $62.4 \%(\mathrm{n}=$ 424) included any antipsychotic medication compliance discussion ( $58 \%$ for $\mathrm{SZ}, 64 \%$ for $\mathrm{BD}$, and $63 \%$ for MDD cohorts; $\mathrm{p}=0.403$ ). Among charts containing discussions with specific terms, only $14.5 \%$ had non-medication treatment discussions and the SZ cohort had the least $(7 \%$; $\mathrm{p}=$ 0.009). However, among charts containing discussions with non-specific terms, the frequency of non-medication treatment discussions increased to $42 \%$ overall (by cohort: $55 \%$ for MDD, $48 \%$ for $\mathrm{SZ}$, and 35\% for BD cohorts; $\mathrm{p}=$ $0.002)$.

In a logistic regression analysis, adjustments were made for patient age, sex, geographic region, baseline medication use, baseline BH utilization, and days from index to each abstracted visit, to identify any differences in odds of a compliance discussion regarding antipsychotic 


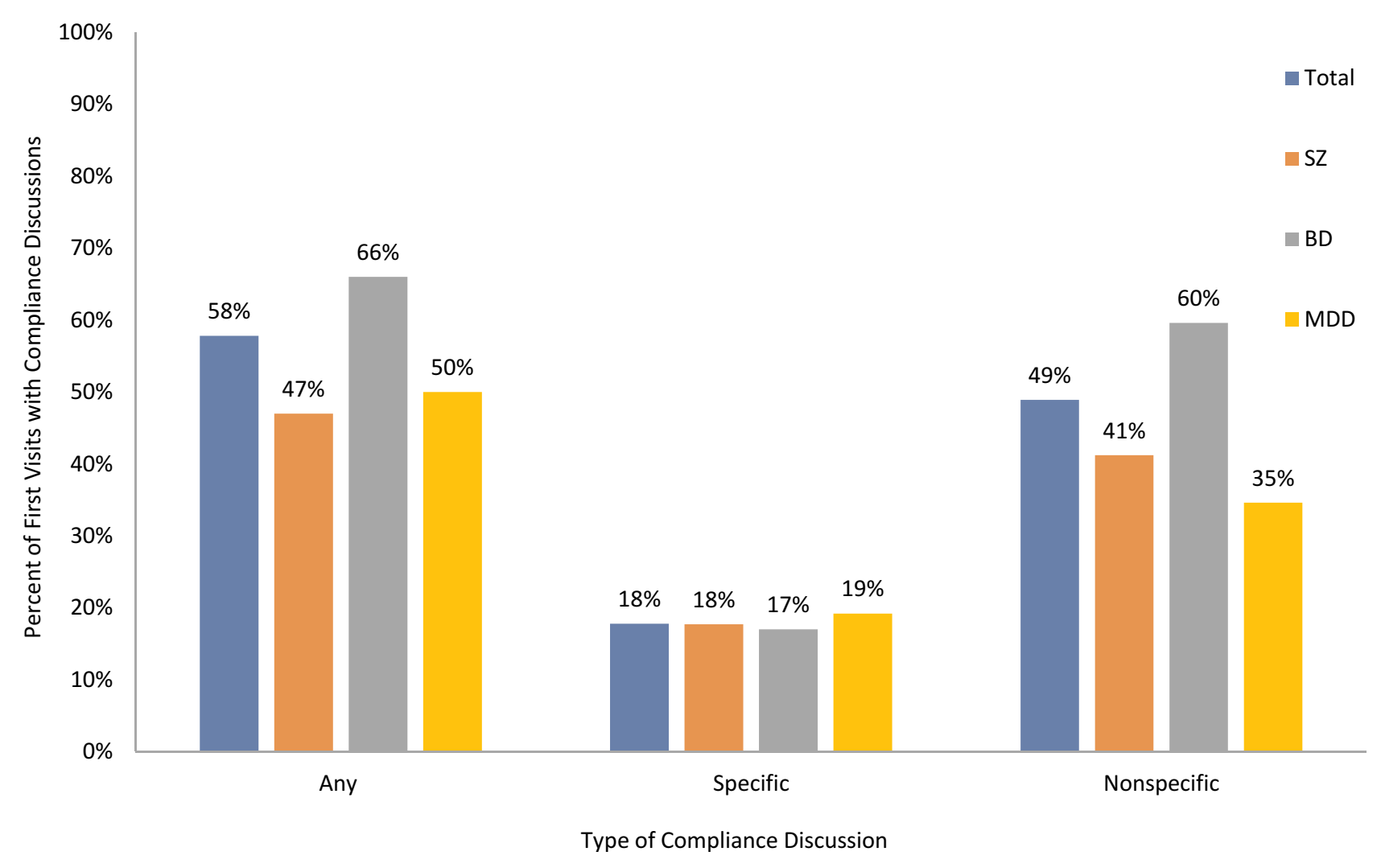

Figure 4 Antipsychotic medication compliance discussions at first visit*.

Notes: *The p-values for all comparisons showed statistically non-significant differences among cohorts.

Abbreviations: BD, bipolar disorder; $\mathrm{BH}$, behavioral health; MDD, major depressive disorder; SZ, schizophrenia.

medication. Regardless of the cohort designation (because all cohorts had evidence of multiple SMI diagnoses), odds of a compliance discussion were lowest among patients with diagnoses of schizophrenia $(\mathrm{OR}=0.562)$ and higher among patients with diagnoses for bipolar disorder $(\mathrm{OR}=$ $1.440)$ or major depressive disorder $(\mathrm{OR}=1.514)(\mathrm{p}>$ 0.05 for all; Table 4).

\section{Discussion}

In the field of severe mental illness, poor patient adherence to antipsychotic medications has been clearly associated with adverse outcomes. Assuming a positive response to patient-provider discussions regarding compliance would improve outcomes, characterizing these discussions in current practice is a vital starting place to develop interventions. This study included patients obtaining outpatient care following an acute inpatient or emergency event, providing healthcare contact points at which compliance discussions should occur. However, among the visits for which charts were abstracted, such discussions did not occur as frequently as expected.
First-visit abstraction revealed $58 \%$ of the entire sample had any discussion addressing compliance with antipsychotic medication, with no significant differences among cohorts. However, using different terminology to identify these discussions was revealing. Fewer than 1 in 5 patients had compliance discussions using the specific terms "compliance," "persistence," or "adherence," but half had evidence of compliance discussions identified using general terms. When all follow-up visits were analyzed, such variation in evidence of compliance discussions by terminology continued. Terminology was similarly a factor in identifying discussions about nonmedication treatments, such as some form of talk therapy. Thus, for future analysis, the language used to define a compliance discussion is critical to obtaining the best understanding. Otherwise, one could postulate that lack of evidence by specific terminology actually indicates lack of any counseling regarding compliance.

Patients' current clinical circumstances were noteworthy: a change in symptoms was documented reason for outpatient visits among two-thirds of the first visits, 


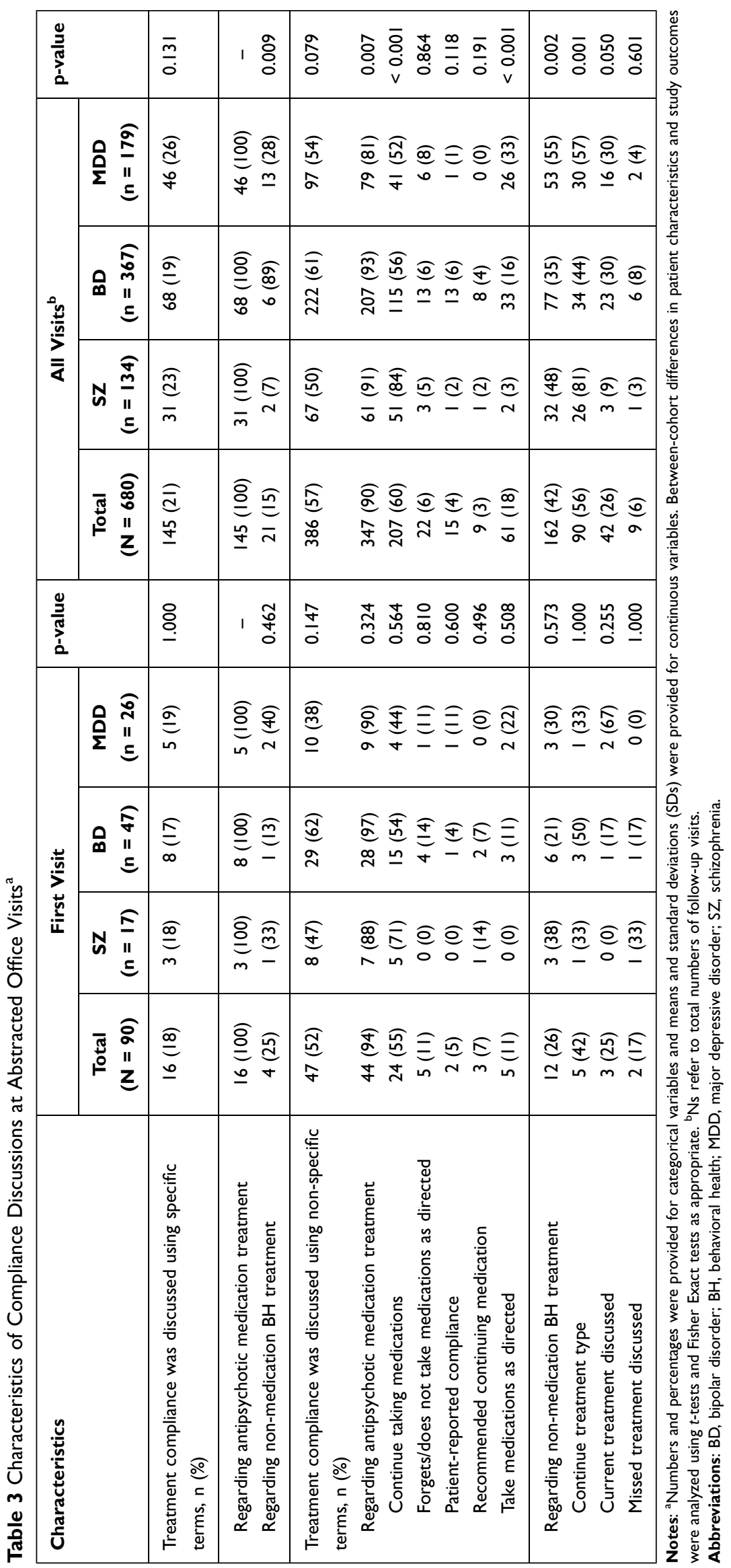


Table 4 Generalized Linear Model for Odds of a Compliance-Related Discussion Regarding Antipsychotic Medication

\begin{tabular}{|c|c|c|c|}
\hline Independent Variables & Odds Ratio & $95 \% \mathrm{Cl}$ & p-value \\
\hline Intercept & - & - & 0.289 \\
\hline Age & 0.994 & $0.967-1.021$ & 0.640 \\
\hline \multicolumn{4}{|l|}{ Sex } \\
\hline Male & ref. & - & - \\
\hline Female & 1.145 & $0.513-2.552$ & 0.741 \\
\hline \multicolumn{4}{|l|}{ Geographic region } \\
\hline Northeast and Midwest & ref. & - & - \\
\hline South & 2.486 & $1.260-4.905$ & 0.009 \\
\hline West & 2.417 & $0.599-9.757$ & 0.215 \\
\hline \multicolumn{4}{|l|}{ 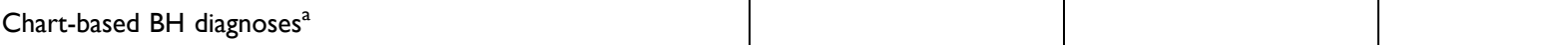 } \\
\hline Schizophrenia & 0.562 & $0.174-1.821$ & 0.337 \\
\hline Bipolar disorder & 1.440 & $0.363-5.707$ & 0.604 \\
\hline Major depression & 1.514 & $0.410-5.595$ & 0.534 \\
\hline \multicolumn{4}{|l|}{ Claims-based baseline characteristics } \\
\hline Evidence of benzodiazepine use & 1.013 & $0.414-2.478$ & 0.978 \\
\hline Number of unique $\mathrm{BH}$ diagnoses ${ }^{\mathrm{b}}$ & 0.919 & $0.582-1.453$ & 0.718 \\
\hline Number of unique medications & 0.962 & $0.888-1.042$ & 0.337 \\
\hline Number of unique behavioral health medications & 0.845 & $0.642-1.111$ & 0.227 \\
\hline Count of $\mathrm{BH}$-related outpatient visits ${ }^{\mathrm{c}}$ & 1.071 & $0.898-1.277$ & 0.447 \\
\hline Total BH-related emergency room costs ${ }^{c}$ & 0.999 & $0.998-1.000$ & 0.020 \\
\hline Number of days between the index event and each office visit & 1.000 & $0.999-1.002$ & 0.553 \\
\hline
\end{tabular}

Notes: Observations read $=680$, observations used $=680$. ${ }^{a}$ These diagnoses do not represent cohorts, but individual diagnoses as a binary $(\mathrm{y} / \mathrm{n})$ variable. ${ }^{\mathrm{b}} \mathrm{BH}$-related conditions include adjustment disorder, anxiety disorder, attention-deficit/hyperactivity disorder, bipolar disorder, depressive disorder, personality disorder, and schizophrenia. ${ }^{\mathrm{B}} \mathrm{BH}-\mathrm{related}$ healthcare resource utilization was defined as any claim with an ICD-9-CM/ICD-I0-CM diagnosis code for BH, excluding cognitive disorders (dementia and Alzheimer disease) and developmental disorders.

Abbreviations: $\mathrm{BH}$, behavioral health; ref., reference.

and medication regimen was changed at half of first visits. With fluctuation in symptoms and medication changes affecting so many of the patients at the first visit, one would expect to observe medication compliance discussions at all first visits. Among all visits, even using nonspecific terms, compliance discussions were observed least among the SZ cohort. Furthermore, although no significant differences were identified by GLM, the odds of a antipsychotic compliance discussion were lowest among patients with a diagnosis of schizophrenia. This is counterintuitive because medication non-adherence has often been demonstrated to be highest among patients with schizophrenia. ${ }^{10}$

A possible reason for surprisingly infrequent compliance discussions is that patients were not newly diagnosed or treatment-naïve and thus were likely counseled previously about compliance. They may have treatmentresistant psychosis or have experienced adverse events with prior treatments, or for other reasons, were resistant to discussing compliance. ${ }^{26,34,35}$ With a relatively short study period, understanding the course of disease for each patient is limited. However, most patients had baseline BH-related claims for ambulatory care, and nearly half for ED or inpatient care. Prior to the index event, they had been prescribed 3 or more unique BH medications and their healthcare burden was substantial before and after the index event. We purposefully selected a sample of patients with potentially severe symptoms, where adherence to treatment may have been challenging and contributed to the acute event that defined the index date. Among such patients, establishing therapeutic alliance and trust between patients and providers is especially important. 23,25

Another notable finding was that first post-index visits occurred more than 1 month after acute event discharge. Although NCQA HEDIS $2020^{36}$ guidelines support follow-up within 30 days, Velligan et al observed up to $40 \%$ of patients with SMI do not have outpatient visits within 30 days of discharge. ${ }^{23}$ Beadles et al $(2015)^{37}$ demonstrated that outpatient follow-up within 30 days was 
associated with increased medication use and ongoing outpatient care utilization. However, first-visit timing may not be influential in this study, because visits within 7 days or led by a different provider than ongoing visits, were not captured. Clinical guidelines suggest all patients should be instructed to return for regular medical management and referred to other treatments, such as individual and group therapy. ${ }^{33}$ Yet, $25 \%$ of first abstracted visits had no evidence of specific instructions to return and half had no evidence of non-pharmacologic BH therapy discussions.

To our knowledge, this is the first study of patient charts for evidence of discussions about compliance with antipsychotic medication. Although our sample size of patients was relatively small, nearly 700 behavioral health outpatient visits were characterized. The sample included a large proportion of patients with more than one $\mathrm{BH}$ diagnosis and highly prevalent anxiety disorder diagnoses. At first visit, two-thirds of charts identified psychosocial stress, which is among several important influential factors in nonadherence. ${ }^{10}$ Thus, despite small sample sizes, our data suggest these were very vulnerable patients with complex diagnoses and treatments - those for whom antipsychotic compliance is notoriously poor-and they were not receiving consistent counseling about compliance.

\section{Study Limitations}

The findings of this study should be considered in view of limitations of observational studies with small sample sizes. Although approximately one-third of eligible medical charts were procured and abstracted, it is not known whether available information regarding compliance discussions differed by providers' choice to participate. Furthermore, we included only patients with continuous coverage by commercial insurance, whose treatment compliance experiences may not be generalizable to patients with other types of coverage or the uninsured.

Although all available $\mathrm{BH}$ visits in each medical chart were abstracted, $\mathrm{BH}$ treatment provided by alternative clinicians was not included. Patients may have been excluded because claims would not have identified a first post-index visit if the service was bundled into the acute event. Restricting the sampling approach may have limited the variance in the study sample, making it difficult to detect differences.

Compliance communication descriptions varied widely among the charts procured, being derived from hand- written progress notes and/or standard fields obtained via electronic medical record software. A standard instruction field, such as "take medications as directed," may not document an oral communication regarding treatment compliance. Moreover, discussions may have taken place that were not documented at all. In either case, accurately describing antipsychotic medication compliance discussions is a challenge for BH-related healthcare for which adherence is already known to be problematic. Finally, the sample size was small, yet the 90 included patients had nearly $700 \mathrm{BH}-$ related visits for analysis.

\section{Conclusions}

Our findings suggest that the frequency of treatment compliance discussions should be increased within ambulatory care visits after an acute inpatient or ED event, particularly for patients with schizophrenia and bipolar disorder. These visits appear to be points at which individualized intervention might affect utilization and cost outcomes. Additionally, these results may represent a call to action to increase focus on the value of the patient and provider collaboration in educational content to reinforce the value of engaging in and documenting these interactions. Increased focus on the partnership of medical management with other forms of treatment may also improve the quality of care.

\section{Acknowledgments}

We gratefully acknowledge Breanna Essoi of Optum and the Clinical Research Team at CIOX Health for their work in obtaining chart abstraction data. Medical writing services were provided by Yvette Edmonds and Caroline Jennermann, employees of Optum, as contracted by Otsuka.

\section{Disclosure}

This study was supported by Otsuka Pharmaceutical Development \& Commercialization, Inc. At the time the study was conducted, authors FF and HW were employees of Otsuka, and authors $\mathrm{CM}$, EK, JW, and $\mathrm{AB}$ were employees of Optum. The authors declare no other conflicts of interest.

\section{References}

1. Mokdad AH, Ballestros K, Echko M, et al. The state of US health, 1990-2016: burden of diseases, injuries, and risk factors among US states. JAMA. 2018;319:1444-1472. doi:10.1001/jama.2018.0158

2. Bloom DE, Cafiero ET, Jané-Llopis E, et al. The global economic burden of noncommunicable diseases. Geneva, Switzerland: World Economic Forum; 2011. Available from: https:/www.weforum.org/ reports/global-economic-burden-non-communicable-diseases.

Accessed May 29, 2020. 
3. National Institute of Mental Health. Mental health information statistics; 2020. Bethesda, MD: NIH. Available from: https://www.nimh.nih.gov/ health/statistics/mental-illness.shtml. Accessed May 29, 2020.

4. Greenberg PE, Fournier AA, Sisitsky T, Pike CT, Kessler RC. The economic burden of adults with major depressive disorder in the United States (2005 and 2010). J Clin Psychiatry. 2015;76:155-162. doi:10.4088/JCP.14m09298

5. Cloutier M, Aigbogun MS, Guerin A, et al. The economic burden of schizophrenia in the United States in 2013. J Clin Psychiatry. 2016;77:764-771. doi:10.4088/JCP.15m10278

6. Cloutier M, Greene M, Guerin A, Touya M, Wu E. The economic burden of bipolar I disorder in the United States in 2015. J Affect Disord. 2018;226:45-51. doi:10.1016/j.jad.2017.09.011

7. Dixon LB, Holoshitz Y, Nossel I. Treatment engagement of individuals experiencing mental illness: review and update. World Psychiatry. 2016;15:13-20. doi:10.1002/wps.20306

8. Easter A, Pollock M, Pope LG, Wisdom JP, Smith TE. Perspectives of treatment providers and clients with serious mental illness regarding effective therapeutic relationships. $J$ Behav Health Serv Res. 2016;43:341-353. doi:10.1007/s11414-015-9492-5

9. Thompson L, McCabe R. The effect of clinician-patient alliance and communication on treatment adherence in mental health care: a systematic review. BMC Psychiatry. 2012;12:87. doi:10.1186/ 1471-244X-12-87

10. Semahegn A, Torpey K, Manu A, Assefa N, Tesfaye G, Ankomah A. Psychotropic medication non-adherence and its associated factors among patients with major psychiatric disorders: a systematic review and meta-analysis. Syst Rev. 2020;9:17. doi:10.1186/s13643-020-1274-3

11. Desai R, Nayak R. Effects of medication nonadherence and comorbidity on health resource utilization in schizophrenia. J Manag Care Spec Pharm. 2019;25:37-44. doi:10.18553/jmcp.2019.25.1.037

12. Forma F, Green T, Kim S, Teigland C. Antipsychotic medication adherence and healthcare services utilization in two cohorts of patients with serious mental illness. Clinicoecon Outcomes Res. 2020;12:123-132. doi:10.2147/CEOR.S231000

13. Higashi K, Medic G, Littlewood KJ, Diez T, Granström O, De Hert M. Medication adherence in schizophrenia: factors influencing adherence and consequences of nonadherence, a systematic literature review. Ther Adv Psychopharmacol. 2013;3:200-218. doi:10.1177/ 2045125312474019

14. Jawad I, Watson S, Haddad PM, Talbot PS, McAllister-Williams RH. Medication nonadherence in bipolar disorder: a narrative review. Ther Adv Psychopharmacol. 2018;8:349-363. doi:10.1177/ 2045125318804364

15. Jiang Y, Ni W. Estimating the impact of adherence to and persistence with atypical antipsychotic therapy on health care costs and risk of hospitalization. Pharmacotherapy. 2015;35:813-822. doi:10.1002/ phar.1634

16. Hong J, Reed C, Novick D, Haro JM, Aguado J. Clinical and economic consequences of medication non-adherence in the treatment of patients with a manic/mixed episode of bipolar disorder: results from the European Mania in Bipolar Longitudinal Evaluation of Medication (EMBLEM) study. Psychiatry Res. 2011;190:110-114. doi:10.1016/j.psychres.2011.04.016

17. Novick D, Haro JM, Suarez D, Perez V, Dittmann RW, Haddad PM. Predictors and clinical consequences of non-adherence with antipsychotic medication in the outpatient treatment of schizophrenia. Psychiatry Res. 2010;176:109-113. doi:10.1016/j.psychres.2009.05.004

18. Offord S, Lin J, Mirski D, Wong B. Impact of early nonadherence to oral antipsychotics on clinical and economic outcomes among patients with schizophrenia. Adv Ther. 2013;30:286-297. doi:10.1007/s12325-013-0016-5
19. De Las Cuevas C, de Leon J, Peñate W, Betancort M. Factors influencing adherence to psychopharmacological medications in psychiatric patients: a structural equation modeling approach. Patient Prefer Adher. 2017;11:681-690. doi:10.2147/PPA.S133513

20. Chapman SC, Horne R. Medication nonadherence and psychiatry. Curr Opin Psychiatry. 2013;26:446-452. doi:10.1097/ YCO.0b013e3283642da4

21. Velligan DI, Sajatovic M, Hatch A, Kramata P, Docherty JP. Why do psychiatric patients stop antipsychotic medication? A systematic review of reasons for nonadherence to medication in patients with serious mental illness. Patient Prefer Adher. 2017;11:449-468. doi:10.2147/PPA.S124658

22. Mago R. Adverse effects of psychotropic medications: a call to action. Psychiatr Clin North Am. 2016;39:361-373. doi:10.1016/j. psc.2016.04.005

23. Velligan DI, Fredrick MM, Sierra C, et al. Engagement-focused care during transitions from inpatient and emergency psychiatric facilities. Patient Prefer Adher. 2017;11:919-928. doi:10.2147/PPA.S132339

24. Lakdawala PD. Doctor-patient relationship in psychiatry. Mens Sana Monogr. 2015;13:82-90. doi:10.4103/0973-1229.153308

25. Chakrabarti S. Treatment alliance and adherence in bipolar disorder. World J Psychiatry. 2018;8:114-124. doi:10.5498/wjp.v8.i5.114

26. Tessier A, Boyer L, Husky M, Bayle F, Llorca PM, Misdrahi D. Medication adherence in schizophrenia: the role of insight, therapeutic alliance, and perceived trauma associated with psychiatric care. Psychiatry Res. 2017;257:315-321. doi:10.1016/j. psychres.2017.07.063

27. Kreyenbuhl J, Record EJ, Palmer-Bacon J. A review of behavioral tailoring strategies for improving medication adherence in serious mental illness. Dialogues Clin Neurosci. 2016;18:191-201. doi:10.31887/DCNS.2016.18.2/jkreyenbuhl

28. Velligan DI, Weiden PJ, Sajatovic M, et al.; Expert Consensus Panel on Adherence Problems in Serious and Persistent Mental Illness. The expert consensus guideline series: adherence problems in patients with serious and persistent mental illness. $J$ Clin Psychiatry. 2009;70(Suppl 4):1-46. doi:10.4088/JCP.7090su1cj

29. Census regions and divisions of the United States; 2020. Available from: https://www2.census.gov/programs-surveys/sahie/referencemaps/2017/us-regdiv.pdf. Accessed May 29, 2020.

30. Clinical Classifications Software (CCS). Clinical Classifications Software (CCS) for ICD-9-CM/ICD-10/CM; 2020. Rockville, MD: Agency for Healthcare Research and Quality. Available from: http:// www.hcup-us.ahrq.gov/toolssoftware/ccs/ccs.jsp. Accessed May 29, 2020.

31. Quan H, Li B, Couris CM, et al. Updating and validating the Charlson Comorbidity Index and Score for risk adjustment in hospital discharge abstracts using data from 6 countries. Am J Epidemiol. 2011;173:676-682. doi:10.1093/aje/kwq433

32. US Department of Labor, Bureau of Labor Statistics. Consumer price index. Medical care. Series ID: SUUR0000SAM; 2017. Available from: http://data.bls.gov/cgi-bin/surveymost?su.2018. Accessed May 29, 2020.

33. APA Guideline Writing Group. American Psychiatric Association practice guideline for the treatment of patients with schizophrenia. Copyright 2019, APA; 2020. Washington, DC: APA. Available from: https://www.psychiatry.org/psychiatrists/practice/clinical-practiceguidelines/review-draft-guidelines. Accessed May 29, 2020.

34. Takeuchi H, Siu C, Remington G, et al. Does relapse contribute to treatment resistance? Antipsychotic response in first-vs. second-episode schizophrenia. Neuropsychopharmacol. 2019;44: 1036-1042. doi:10.1038/s41386-018-0278-3

35. Bozzatello P, Bellino S, Rocca P. Predictive factors of treatment resistance in first episode of psychosis: a systematic review. Front Psychiatry. 2019;10:67. doi:10.3389/fpsyt.2019.00067 
36. National Committee for Quality Assurance. Draft document for HEDIS Public Comment: HEDIS health plan follow-up after hospitalization for mental illness (FUH) measure; 2020. Washington, DC: NCQA. Available from: https://www.ncqa.org/hedis/measures/follow-up-afterhospitalization-for-mental-illness/. Accessed May 29, 2020.
37. Beadles C, Ellis AR, Lichstein JC, et al. First outpatient follow-up after psychiatric hospitalization: does one size fit all? Psychiatric Serv. 2015;66:364-372. doi:10.1176/appi.ps.201400081

\section{Publish your work in this journal}

Pragmatic and Observational Research is an international, peerreviewed, open access journal that publishes data from studies designed to reflect more closely medical interventions in realworld clinical practice compared with classical randomized controlled trials (RCTs). The manuscript management system is completely online and includes a very quick and fair peer-review system. Visit $\mathrm{http}: / / \mathrm{www}$.dovepress.com/testimonials.php to read real quotes from published authors. 